\title{
ECG and EDA Information Transfer on Emotion Evaluation ${ }^{\dagger}$
}

\author{
Luísa Castro ${ }^{1,2}$, Filipa Barros ${ }^{3}$, Sandra C. Soares ${ }^{3}$ and Susana Brás ${ }^{4,5}$ \\ 1 INESC TEC - Institute for Systems and Computer Engineering, Technology and Science, Engineering \\ Faculty, University of Porto, Porto, Portugal \\ 2 Center for Health Technology and Services Research, Porto Medical School, University of Porto, Porto, \\ Portugal \\ 3 William James Center for Research, DEP, University of Aveiro, Aveiro, Portugal \\ 4 IEETA - Institute of Electronics and Informatics Engineering of Aveiro, University of Aveiro, Aveiro, \\ Portugal \\ 5 DETI, University of Aveiro, Aveiro, Portugal \\ + Presented at the Entropy 2021: The Scientific Tool of the 21st Century, 5-7 May 2021; Available online: \\ https://sciforum.net/conference/Entropy2021/.
}

Published: 5 May 2021

Emotions are behind decision-making, perception and learning. Studying emotions and their responses allow us to understand people's preferences and their strategies to adapt across contexts. Both peripheral and central nervous systems are activated by emotions, which are translated on behavioural and physiological alterations. Usually, the ecological validity of emotion induction studies is truly compromised due to the multiple electrodes used in the experiment, affecting the usual participants' daily routine. The major idea of this study is to compare signals in emotion quantification, to select the optimal data collection setup. In this work, we targeted two physiological signals: electrocardiogram (ECG), and electrodermal activity (EDA) in the happy condition.

Data from 4 participants were collected in healthy volunteers, which came to the lab three times. Each session intended to induce one emotion between happy, fear, neutral. At the beginning, participants rested for 4 minutes to collect baseline data. Afterwards, they watched intense movies associated with each condition for 25 minutes.

In this work, it is intended to study the information transfer between ECG and EDA, for the baseline and happy condition. The information dynamics based on the linear Gaussian approximation was computed to characterize information storage and transfer between ECG and EDA signals.

Considering the self-entropy of each signal, EDA presented slightly greater values than ECG. It was observed that in all participants, the ECG transfers information to the EDA, indicating that the ECG information may pertain in the system. Hence, the exploratory results achieved in this study may indicate that the ECG is a stronger signal when we want to evaluate emotions in real contexts. Future studies should address the study of information transfer between these and other physiological signals, and also different emotional conditions. 